\title{
Hacia una taxonomía de los sistemas regionales de innovación en México
}

\section{Towards a taxonomy of regional innovation systems in Mexico}

\author{
Cuitláhuac Valdez-Lafarga \\ Jorge INÉS LEÓN-BALDERRAMA*
}

\begin{abstract}
This paper examines the level of heterogeneity of mexican states regarding their potential and performance as innovation systems. The main objective is the classification of the regional innovation systems (RIS), in the context of the states of the country, based on a series of indicators that correspond to their socialeconomic and institutional conditions, as well as their capacities and innovation results. Cluster analysis shows the existence of six types of RIS in Mexico, with distinctive strengths and weaknesses, in terms of the proposed innovation dimensions.
\end{abstract}

Keywords: regional innovation system, taxonomy, cluster analysis.

\section{Resumen}

En este trabajo se examina el nivel de heterogeneidad de las entidades federativas mexicanas en cuanto a su potencial y desempeño como sistemas de innovación. El objetivo central es la clasificación de los sistemas regionales de innovación (SRI), en el contexto de las entidades federativas, a partir de una serie de indicadores correspondientes a sus condiciones socioeconómicas e institucionales, así como a sus capacidades y resultados en innovación. Mediante análisis de clusters se muestra la existencia de seis tipos de SRI en México, con fortalezas y debilidades distintivas, en términos de las dimensiones de innovación propuestas.

Palabras clave: sistema regional de innovación, taxonomía, análisis de clusters.

* Centro de Investigación en Alimentación y Desarrollo, A. C. Correos-e: cui.valdez@gmail. com, jleon@ciad.mx 


\section{Introducción}

La literatura sobre innovación y sistemas de innovación acumulada desde mediados de la década de los ochenta del siglo pasado destaca la importancia de los procesos de innovación para el desarrollo económico moderno. Si bien una gran parte de los estudios sobre el tema giran en torno a la conceptualización de los sistemas de innovación, incluyendo sus componentes, funciones y naturaleza (Freeman, 1987; Lundvall, 1985; Cooke, 1998; Niosi, 2002; entre otros), los últimos años han visto el surgimiento de estudios enfocados en evaluar el desempeño de dichos sistemas.

A principios de 1990 surgen una serie de indicadores de innovación que complementa a los clásicos de input (entrada) y output (salida) utilizados en procesos de producción económica. Esto se debió en gran parte al proceso llevado a cabo para la publicación del Manual de Oslo (OECD, 1992), así como las subsecuentes encuestas de innovación europeas, Conmmunity Innovation Survey (CIS). Lo anterior permitió estudiar otras dimensiones de innovación mediante análisis cuantitativo, además de las dimensiones técnicas, económicas, sociales e institucionales que se habían estudiado bajo un enfoque cualitativo (Evangelista et al., 1998; Smith, 2005).

Dentro de las líneas de estudio alrededor de la innovación con enfoque cuantitativo se encuentra la clasificación taxonómica de los procesos/ sistemas de innovación. Uno de los trabajos pioneros que propuso el concepto de las taxonomías enfocadas en los procesos de cambio tecnológico e innovación es el estudio seminal de Pavitt (1984), cuyo objetivo general era describir el comportamiento de las empresas innovadoras para predecir sus acciones y sugerir un marco para el análisis de políticas de innovación. A este estudio le seguiría un amplio cúmulo de trabajos que buscaron complementarlo o ampliarlo e incluso mejorarlo.

Las taxonomías son una herramienta de gran utilidad para la clasificación de fenómenos, ya que buscan maximizar las diferencias entre grupos y tienen la capacidad de reducir la complejidad de la población bajo estudio a macro-clases fácilmente recordables (Archibugi, 2001). En las ciencias sociales encontramos el enfoque empírico y el teórico como los dos enfoques generales de clasificación (Warriner, 1984; Doty y Glick, 1994). Los estudios sobre la innovación con el objetivo de clasificar, ya sea la innovación misma o los procesos innovadores, se basan en el enfoque empírico, el cual comienza con la recopilación de datos para las entidades bajo estudio, los cuales son procesados mediante métodos estadísticos para producir grupos a partir de las medidas de similitud y las técnicas estadísticas utilizadas (Coccia, 2006; Capello y Lenzi, 2013; Reslinger, 2014). 
Algunos trabajos de investigación basados en este último enfoque (Balzat y Pyka, 2006; Godinho et al., 2006; Pinto y Santos, 2013, por ejemplo) han propuesto una taxonomía de sistemas de innovación, particularmente, sistemas nacionales de innovación. Sin embargo, una de las limitantes que observamos para estos estudios es la incapacidad para explicar las diferencias en el desempeño hacia el interior de los países. Esto resulta especialmente relevante en naciones en vías de desarrollo que muestran diferencias marcadas en términos de crecimiento económico de algunas regiones al nivel subnacional. Tal es el caso mexicano, el cual se ha caracterizado por diferencias significativas en el desarrollo de su región norte, centro y sur. Sin embargo, para realizar el análisis al nivel subnacional se requiere un marco conceptual para sistemas de innovación diferente al ámbito nacional y la alternativa reside en los SRI.

Existen similitudes entre un sistema nacional y uno regional, ya que de manera general se cuentan con los mismos elementos y las mismas funciones básicas descritas para cualquier sistema de innovación; (1) un subsistema estructural de explotación del conocimiento (principalmente empresas); (2) un subsistema de generación de conocimiento (universidades, centros de investigación, etc.); (3) un contexto institucional (normas, confianza, rutinas, etc.) donde se generan las interacciones del sistema (Cooke et al., 1998).

En ese sentido, el concepto de SRI puede entenderse como una sección del sistema nacional de innovación, donde las características principales permanecen válidas al estudiar áreas más pequeñas (Buesa et al., 2006). Una observación importante hecha por Asheim y Cooke (1999) es que el concepto de región resalta un nivel importante de gobernanza de los procesos económicos entre el nivel nacional y el local o municipal, esto es el nivel estatal. Además, Asheim y Coenen (2005) señalan la importancia del nivel meso de las naciones como base de coordinación económica, aunque la forma de administrar estas actividades puede diferir significativamente entre países.

Por lo anterior, para el caso mexicano consideramos que es pertinente tomar las entidades federativas como un modelo de SRI que nos permita llevar a cabo un estudio empírico sobre las diferencias y similitudes en las características de los sistemas estatales de innovación en México, a partir de sus dimensiones. La motivación del estudio es contribuir a la comprensión de las actividades de ciencia, tecnología e innovación en México, desde una perspectiva regional y un enfoque cuantitativo. El objetivo es construir una taxonomía mediante análisis multivariado de datos que permita: (1) diferenciar los SRI con base en las características de condiciones, capacidades y desempeño del sistema, y (2) clasificar los SRI de acuerdo con características particulares que los definen. 
La estructura de este documento es la siguiente. La sección 1 presenta un marco conceptual donde se aborda el uso del enfoque de sistema regional de innovación como marco analítico. La sección 2 muestra una breve revisión de antecedentes para estudios taxonómicos enfocados en sistemas de innovación. La sección 3 expone la metodología empleada para el estudio, con una breve descripción de los métodos y herramientas utilizadas. La sección 4 consiste en resultados y discusión para el análisis de clusters realizado, así como la clasificación propuesta a partir de estos resultados. Finalmente, las conclusiones en donde se plantean las reflexiones sobre la aportación del estudio y los posibles enfoques para futuras investigaciones.

\section{Los sistemas regionales de innovación como marco de análisis}

No existe una definición universalmente aceptada para el concepto de SRI; sin embargo, existe cierto grado de convergencia entre las diferentes propuestas del concepto, lo cual incluye la existencia de una red localizada de actores e instituciones públicas y privadas que interaccionan para generar, transferir, modificar y difundir conocimiento y nuevas tecnologías, dentro de la estructura de producción de una región (Cooke,1992; Doloreux, 2003; Asheim y Gertler, 2006; Capello y Lenzi, 2013).

$\mathrm{El}$ argumento central es que este conjunto de actores e instituciones producen efectos penetrantes y sistémicos que impulsan a las compañías dentro de la región a desarrollar formas específicas de capital, derivadas de relaciones sociales, normas, valores e interacciones dentro de la comunidad, para reforzar la capacidad y competitividad regional en innovación (Gertler et al., 2000).

Existen varios argumentos a favor del enfoque regional para el estudio de la innovación. El principal es que la aglomeración regional proporciona el mejor contexto para una economía del conocimiento basada en la innovación (Hudson, 1999), para la creación y difusión del conocimiento así como para el aprendizaje. Doloreux y Parto (2004) mencionan que la popularidad del enfoque se debe a que proporciona una narrativa sobre las dimensiones intangibles de desarrollo económico local y los procesos de circulación del conocimiento y aprendizaje en una escala regional aparentemente más manejable.

En términos de los factores que producirán el efecto de derrama de conocimiento (la homogeneidad en las condiciones sociales, culturales e institucionales), el enfoque de los SRI visualiza a la nación como la composición de regiones no-homogéneas, y argumenta que cada región debe tener su propio sistema de gobernanza (Jang, 2006). Se ha mostrado empíricamente que las regiones pueden contener grandes secciones de 
una cadena de valor (por ejemplo, los distritos industriales italianos), así como una estructura gubernamental relativamente autónoma (entidades federativas como Baden-Württemberg en Alemania, o Catalaluña en España) (Asheim y Coenen, 2005).

Concretamente, el uso del SRI como marco conceptual consta de dos conjuntos de estudios: a) empíricos comparativos de varias regiones y b) de sistemas individuales. Los comparativos tienen como objetivo describir el funcionamiento de los SRI para encontrar factores y mecanismos deseables para promover la competitividad y la innovación, así como evaluar las implicaciones de las políticas en innovación. Su contribución consiste en proporcionar un estado del arte para la clarificación conceptual de los sistemas regionales a través del enfoque de analizar el impacto de diferentes tipos de SRI en distintos países (Doloreux y Parto, 2004).

El segundo conjunto de estudios consiste en la evaluación de sistemas individuales para determinar hasta qué punto realmente constituyen un sistema de innovación. Su contribución reside en proporcionar una mejor comprensión de la naturaleza y dinámica del desarrollo regional. De esta manera, permiten identificar los factores que contribuyen al surgimiento y sustento de un SRI, las dinámicas sociales e institucionales que apoyan la actividad de innovación de la región, así como la descripción de las diferentes interacciones entre actores y factores dentro de la región (Edquist et al., 2000; Isaksen, 2004; Diez-Revilla, 2002; Cooke et al., 2000). Una contribución fundamental de estos estudios es la evidencia que nos lleva a concluir que no existe un modelo único para generalizar las dinámicas de un SRI exitoso.

Otro tema de discusión importante consiste en la determinación de la unidad de análisis que se considera representativa de un SRI. Sin la intención de profundizar mucho, encontramos diferentes propuestas en la literatura sobre este punto: algunos consideran la ciudad o las zonas metropolitanas como unidades adecuadas para estudiar los procesos de innovación (Crevoisier y Camagni, 2000; Simmie, 2001; Diez-Revilla, 2002; Feldman y Audretsch, 1999). Otros proponen un nivel dentro de la ciudad o zona metropolitana, al cual denominan local (Asheim e Isaksen, 2002; Saxenian, 1994; Porter, 1998; Enright, 2003). También hay quienes utilizan un nivel de mayor agregación que, aunque diverso en su conformación política para diferentes países, utiliza una escala supraregional/subnacional.

Tal es el caso de estudios sobre las provincias canadienses de Ontario (Gertler y Wolfe, 1998) y Quebec (Latouche, 1998), la provincia belga de Wallonia (Capron y Cincera, 1998) o regiones portuguesas (Santos y Simóes, 2014). Para el caso de México, su similar serían las entidades federativas, las cuales se han utilizado como modelo de análisis para 
diagnósticos en ciencia, tecnología e innovación realizados por el Foro Consultivo Científico y Tecnológico (ғсСут) para cada estado de la república mexicana (ғсCут, 2010, 2012 y 2014). Similarmente, el estudio Crespi y D'Este (2011) utiliza la entidad federativa mexicana como modelo de SRI.

La razón detrás de adoptar provincias o estados como unidad de análisis reside en que estos niveles generalmente cuentan con estructuras institucionales específicas y tradiciones culturales que facilitan y regulan el comportamiento económico y la actividad social (Gertler y Wolfe, 1998). Por lo tanto, esta unidad territorial puede sostener las características planteadas en el concepto de sistema regional de innovación.

\section{Antecedentes de elaboración de taxonomías para sistemas de innovación}

Una de las formas a través de las cuales se ha venido estudiando cuantitativamente el cambio tecnológico y la innovación ha sido la clasificación taxonómica. Si bien ésta es un proceso común en las ciencias físicas, sociales y de la vida, las taxonomías representan una forma específica de este tipo de organización. El propósito más básico de una taxonomía es maximizar las diferencias entre grupos bajo estudio, mientras que maximiza las similitudes entre los miembros de cada grupo. Esto se logra a través de un proceso basado en reglas sobre cómo formar y representar grupos (taxa) para luego nombrarlos (nomy) (Coccia, 2006). Las ciencias sociales cuentan con la clasificación teórica y la empírica como sus dos enfoques generales de organización.

La clasificación teórica desarrolla un análisis de diferencias a la que se le conoce como tipología. En cambio, la empírica (y la de mayor interés para el presente estudio) produce grupos a través de datos recopilados y procesados mediante métodos estadísticos, de acuerdo con las medidas de similitud calculadas por dichos métodos. Este último enfoque se utiliza para clasificar cuantitativamente. A continuación se presenta una breve revisión de la literatura alrededor de las taxonomías de sistemas de innovación a partir de sus características en condiciones y desempeño.

Los últimos ańos han visto el surgimiento de enfoques para clasificar diferentes formas de organización de mercados, las cuales consideran a las instituciones y su rol en la organización económica (Jacobides y Kudina, 2013; Campbelle y Pedersen, 2014). Uno de los enfoques más importantes es el de variedades de capitalismo propuesto por Hall y Soskice (2001). Aunque inicialmente se propuso como un concepto descriptivo, Amable (2003) ha aplicado el enfoque utilizando técnicas econométricas. El resultado fue una taxonomía del capitalismo para economías 
industrializadas. Un factor importante de las economías capitalistas es la organización de la innovación y su rol en las economías de mercado y su estructura institucional (Balzat y Pyka, 2006). El concepto de sistema de innovación, de manera similar al concepto de variedades de capitalismo, se enfoca en las especificidades de la estructura institucional y las características específicas de su localización.

Motivados por la compatibilidad entre los enfoques de clasificación de mercados y los sistemas de innovación, Balzat y Pyka (2006) realizaron un estudio empírico buscando las similitudes y diferencias estructurales, así como de desempeño en innovación de los sistemas de innovación para una muestra de 18 países de la OCDE; con el fin último de proveer una mejor comprensión de las estructuras específicas de esos países. Por su parte, Godinho et al. (2006) llevaron a cabo un ejercicio exploratorio para examinar los sistemas de innovación a través de ocho dimensiones, para una muestra de 69 países, con la intención de encontrar los principales factores que diferencian a los sistemas nacionales de innovación, permitiendo proponer una taxonomía. Su objetivo era determinar si es posible aplicar el mismo concepto de sistema nacional de innovación a todos los países, independientemente de sus características específicas.

En cuanto a los estudios cuantitativos sobre taxonomías de los SRI se tiene que éstos son muy escasos. La mayoría se han realizado para proporcionar tipologías de regiones europeas, basadas en sus capacidades y desempeño económico y tecnológico. La cantidad limitada de estudios empíricos en este campo se debe en gran parte a la falta de datos adecuados a nivel regional. Esto podría explicar el sesgo en la literatura hacia estudios teóricos y no empíricos (MacKinnon et al., 2002). De hecho, autores como Malmberg y Maskell (1997) han criticado la falta de estudios sobre los SRI que hagan uso de datos agregados para un gran número de regiones.

Existen dos enfoques para la obtención de tipologías de los SRI, el primero hace uso de casos de estudio de manera iterativa para ir comprobando trabajos conceptuales previos. De esta manera, encontramos que Cooke (1998) combina tres tipos de gobernanza de los SRI: desde abajo, en red e intervencionista; con tres dimensiones de innovación empresarial: localista, interactiva, globalizada, para un total de nueve tipos de SRI. Asheim (2007) propone tres tipos de SRI: alojados territorialmente, regionalmente encadenados y nacionalmente regionalizados.

El segundo enfoque para encontrar tipologías se basa en análisis estadísticos para un conjunto de regiones. El cuadro 1 muestra un resumen de los estudios europeos más relevantes en esta área. Es importante notar que el tipo de variables y conceptos utilizados por los investigadores de 


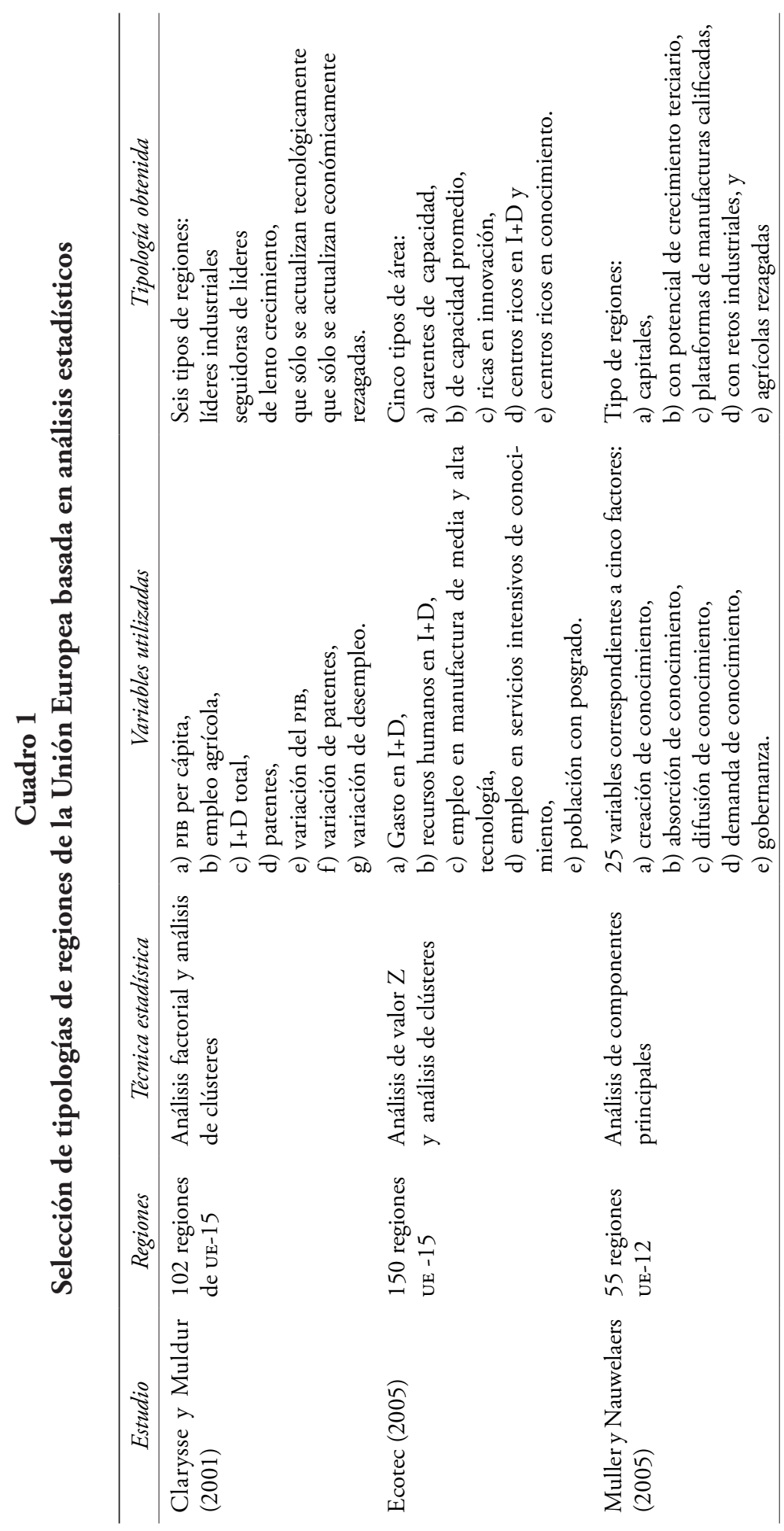




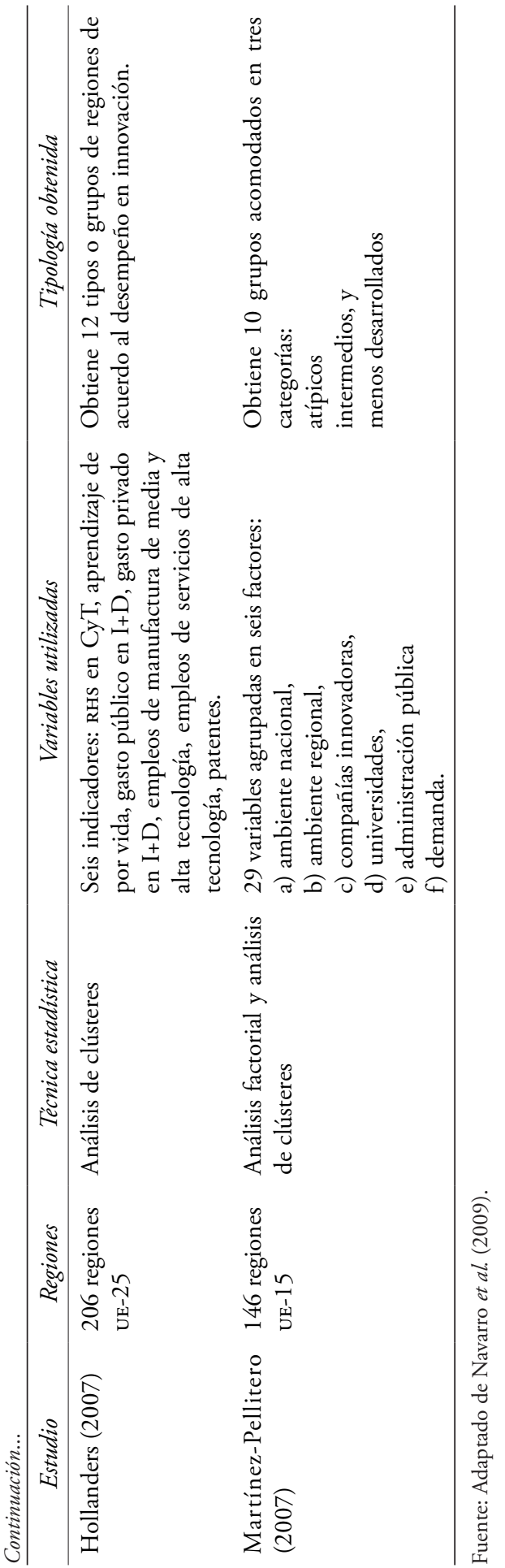


los SRI en sus análisis estadísticos está altamente influenciado por la limitada disponibilidad de datos a nivel regional (Navarro et al., 2009).

Como puede observarse la mayoría de los estudios que se han realizado para la Unión Europea se basan en muestras grandes de regiones, tomando como unidad de análisis las mismas unidades administrativas de esta organización. Otra característica es el uso recurrente de métodos estadísticos de análisis multivariado, especialmente el análisis de cluster.

De manera significativa destaca que los esquemas de análisis que fundamentan la selección de los indicadores particulares ponen énfasis en las capacidades e insumos de ciencia y tecnología, especialmente los recursos públicos destinados a la educación y la investigación y desarrollo. En cuanto a las topologías obtenidas, las formas de caracterización son muy variadas, puesto que, mientras que algunas se centran en la dimensión tecnológica, otras, lo hacen en el nivel de desarrollo de los SRI, el estado de avance tecnológico o la estructura productiva (especialización económica).

Por otra parte, persiste una notable carencia de estudios sobre la situación en las naciones en desarrollo. Esto puede deberse, en parte, a las limitaciones de disponibilidad de fuentes de datos adecuados para el análisis de la innovación, sus componentes y condicionantes a nivel regional, especialmente en los países de América Latina. Sólo recientemente, Crespi y D'Este (2011), utilizando el análisis de cluster, proponen una clasificación de los sRI para el caso de América Latina, específicamente las entidades federativas de Brasil, Chile, Colombia y México.

Un enfoque alternativo que pudiera ser de utilidad para superar la limitación de los enfoques de clasificación de los SRI basados exclusiva o predominantemente en las capacidades de CyT y los recursos para la I+D, es el propuesto por Godinho et al. (2006), quienes se enfocan en cuatro grandes aspectos de los sistemas de innovación que atienden capacidades, condiciones y resultados: $a$ ) precondiciones para la innovación; $b$ ) insumos del sistema; $c$ ) condiciones estructurales $\mathrm{y}, d$ ) resultados del sistema.

\section{Información y métodos utilizados para la elaboración de una taxonomía de los SRI en México}

La propuesta de taxonomía de los SRI presentada en este trabajo parte de sistematizar las diferencias y factores comunes entre sistemas de innovación en el contexto de las entidades federativas de México, a partir de diversos indicadores que tienen que ver con condiciones económicas, sociales, 
institucionales, así como capacidades de innovación y resultados en términos de generación de conocimiento e innovación.

\subsection{Dimensiones e indicadores para la clasificación de los SRI}

Con base en lo anterior, esta investigación se realiza con algunas adecuaciones al modelo analítico de Godinho et al. (2006) en términos de los indicadores utilizados, y de acuerdo a la disponibilidad de datos para éstos. A continuación se describen brevemente las dimensiones e indicadores utilizados en el presente estudio (cuadro 2).

- Precondiciones para la innovación. Se consideran dos clases de precondiciones para la innovación: primero las de mercado y después las institucionales.

- Entradas al sistema. En conexión con las condiciones mencionadas anteriormente, se consideran otras dos clases: "la inversión en intangibles y tangibles" y "el conocimiento científico".

- Características estructurales. Se refiere a la especialización productiva y el grado de internacionalización de la economía y comunicación externa.

- Salidas del sistema. Las principales salidas del sistema tienen que ver naturalmente con el desempeño del SRI, pero de igual forma con la difusión, que es la circulación y diseminación del conocimiento y nuevas tecnologías entre las diferentes partes del sistema.

A continuación se describen cada una de las dimensiones comprendidas en estos cuatro grandes aspectos de los SRI, así como los indicadores particulares que incluye cada dimensión.

Dimensión 1: Condiciones de Mercado. La conceptualización de la innovación implica la comercialización de los productos y servicios generados por la investigación y el desarrollo. Esto significa que parte del éxito de estas innovaciones reside en tener un mercado adecuado en términos de la demanda de los productos y servicios generados. Además, considerando las dificultades que conlleva la distancia geográfica ligadas a costos de transacciones, costos de transporte o la disponibilidad de canales de distribución adecuados, el mercado regional generará las mayores oportunidades para la mayoría de las empresas involucradas en el sistema regional de innovación. La Dimensión 1 busca reflejar la potencialidad de la demanda que existe para las innovaciones generadas en la región, a través de los indicadores de Producto Interno Bruto, Densidad Poblacional e Ingreso Bruto Per Cápita. 
Dimensión 2: Desarrollo institucional. Las instituciones que regulan y dan forma al comportamiento de los agentes económicos dentro del sistema de innovación son difíciles de representar cuantitativamente. Para superar esta limitante se buscó medirlas indirectamente a través de indicadores que pudieran reflejar el efecto de las instituciones sobre la región. De esta manera, los indicadores seleccionados fueron los índices de calidad de transparencia, de efectividad de gobierno y de corrupción y buen gobierno. Mediante estos indicadores se pretende describir las condiciones institucionales generadas por el desempeño de los gobiernos estatales desde la perspectiva de la propia población. Los índices son calculados por organismos independientes como la Conferencia Mexicana para el Acceso a la Información Pública (Comaip), el Centro de Estudios Económicos del Sector Privado, A. C. (CEesp) y Transparencia Mexicana.

Dimensión 3: Inversión en intangibles y capital físico. Existen dos tipos de inversiones primordiales en un sistema de innovación: las inversiones en recursos humanos y en recursos físicos. La primera significa la creación de una masa crítica de generación de ideas, factor crítico para la generación de innovación. Por su parte, la inversión en recursos físicos implica la existencia de infraestructura física apropiada para llevar a cabo actividades de I+D (instituciones de educación superior, centros de investigación públicos y privados, instalaciones de laboratorios con tecnología de punta, etc.). Para representar los esfuerzos en el desarrollo de actividades de ciencia, tecnología e innovación, se contemplan tres indicadores: el gasto en educación como porcentaje del PIB, el gasto en educación per cápita para cada entidad y la inversión del gobierno como porcentaje del presupuesto total del estado dirigido a ciencia, tecnología e innovación. Adicionalmente, se incluye la formación bruta de capital fijo como indicador del valor añadido a la economía que pudiera beneficiar las actividades de innovación.

Dimensión 4: Conocimiento Científico. Esta dimensión busca medir las condiciones existentes para la generación de productos con la potencialidad de convertirse en innovación, así como la orientación hacia una sociedad del conocimiento; con este propósito se seleccionaron cuatro indicadores: número de investigadores en el Sistema Nacional de Investigadores (SNI) como proporción de la PEA, la producción científica per cápita, la población con estudios de posgrado y los centros de investigación públicos y particulares, estos dos últimos como proporción de la población económicamente activa. Los primeros dos indicadores buscan reflejar la potencialidad para generar conocimiento, la orientación hacia actividades relacionadas con ciencia, tecnología e innovación y la producción de conocimiento, el cual tiene el potencial de convertirse en innovación. El 
último indicador busca medir la infraestructura presente en los estados que incide sobre la generación de conocimiento.

\section{Cuadro 2}

\section{Dimensiones e indicadores de los SRI}

\begin{tabular}{|c|c|c|}
\hline Dimensión & Indicadores & Descripción y fuentes \\
\hline \multirow[t]{3}{*}{$\begin{array}{l}1 \text { Condiciones } \\
\text { de mercado }\end{array}$} & PIB & $\begin{array}{l}\text { Producto Interno Bruto en miles de pesos } \\
\text { (transformado con } \log 10) \text {. Inegi (2010) }\end{array}$ \\
\hline & $\begin{array}{l}\text { Densidad de } \\
\text { Población }\end{array}$ & $\begin{array}{l}\text { Densidad poblacional por } \mathrm{km}^{2} \text { (transformado } \\
\text { con } \log 10 \text { ). Inegi (2010) }\end{array}$ \\
\hline & Ingreso Bruto & $\begin{array}{l}\text { Ingreso bruto per cápita en miles de pesos. } \\
\text { Inegi (2010) }\end{array}$ \\
\hline \multirow[t]{3}{*}{$\begin{array}{l}2 \text { Desarrollo } \\
\text { institucional }\end{array}$} & $\begin{array}{l}\text { Calidad de Trans- } \\
\text { parencia }\end{array}$ & $\begin{array}{l}\text { Índice de calidad de transparencia calificada de } \\
\text { 1-100. Comaip (2008) Conferencia Mexicana } \\
\text { para la Información Pública }\end{array}$ \\
\hline & $\begin{array}{l}\text { Efectividad de } \\
\text { Gobierno }\end{array}$ & $\begin{array}{l}\text { Índice de opinión empresarial sobre marco } \\
\text { regulatorio (más alto mejor). CEESP (2008) } \\
\text { Centro de Estudios Económicos del Sector } \\
\text { Privado, A. C. }\end{array}$ \\
\hline & $\begin{array}{l}\text { Índice de Corrup- } \\
\text { ción }\end{array}$ & $\begin{array}{l}\text { Índice de corrupción y buen gobierno (1/Ind). } \\
\text { Transparencia Mexicana (2007) }\end{array}$ \\
\hline \multirow{4}{*}{$\begin{array}{l}3 \text { Inversión en } \\
\text { intangibles y } \\
\text { capital físico }\end{array}$} & FBCF & Formación Bruta de Capital Fijo. Inegi (2008) \\
\hline & $\begin{array}{l}\text { Gasto en educación/ } \\
\text { PIB }\end{array}$ & $\begin{array}{l}\text { Gasto en educación como porcentaje del pIB } \\
\text { (en millones de pesos). CIEP (2011) Centro de } \\
\text { Investigación Económica y Presupuestaria }\end{array}$ \\
\hline & $\begin{array}{l}\text { Gasto en educación } \\
\text { per cápita }\end{array}$ & $\begin{array}{l}\text { Gasto en educación per cápita en millones de } \\
\text { pesos (transformado con log 10). CIEP (2011) }\end{array}$ \\
\hline & $\begin{array}{l}\text { Inversión de } \\
\text { Gobierno }\end{array}$ & $\begin{array}{l}\text { Presupuesto para Ciencia, Tecnología e Inno- } \\
\text { vación como porcentaje del presupuesto total } \\
\text { del estado. FCCyT (2010-2012) }\end{array}$ \\
\hline \multirow[t]{4}{*}{$\begin{array}{l}4 \text { Conoci- } \\
\text { miento } \\
\text { científico }\end{array}$} & $\begin{array}{l}\text { Número de } \\
\text { investigadores }\end{array}$ & $\begin{array}{l}\text { Investigadores SNI de las áreas de Biotecnología, } \\
\text { Físico matemáticas y ciencias de la tierra, me- } \\
\text { dicina y ciencias de la salud, Biología y quími- } \\
\text { ca e Ingenierías, por cada } 10,000 \text { de la PEA. } \\
\text { Conacyt (2011) }\end{array}$ \\
\hline & $\begin{array}{l}\text { Publicaciones per } \\
\text { cápita }\end{array}$ & $\begin{array}{l}\text { Publicaciones per cápita (aproximado). Cona- } \\
\text { cyt (2011) }\end{array}$ \\
\hline & $\begin{array}{l}\text { Población con } \\
\text { estudios de posgrado }\end{array}$ & $\begin{array}{l}\text { Población con estudios de posgrado por cada } \\
1000 \text { de la PEA. Inegi (2010) }\end{array}$ \\
\hline & $\begin{array}{l}\text { Número de centros } \\
\text { de investigación }\end{array}$ & $\begin{array}{l}\text { Centros de investigación (UNAM, IPN, CINVEs- } \\
\text { TAV, Universidades públicas estatales, Conacyt, } \\
\text { Secretarías de estado y otros) por cada } 10,000 \\
\text { de la PEA. Inegi (2010) }\end{array}$ \\
\hline
\end{tabular}


Continuación...

\begin{tabular}{|c|c|c|}
\hline Dimensión & Indicadores & Descripción y fuentes \\
\hline \multirow[t]{3}{*}{$\begin{array}{l}5 \text { Estructura } \\
\text { productiva }\end{array}$} & $\begin{array}{l}\text { Industria de } \\
\text { exportación }\end{array}$ & $\begin{array}{l}\text { Valor agregado de la industria manufacturera, } \\
\text { maquiladora y servicios de exportación (aproxi- } \\
\text { mación). Inegi (2008) }\end{array}$ \\
\hline & $\begin{array}{l}\text { Especialización en } \\
\text { industria manufac- } \\
\text { turera }\end{array}$ & $\begin{array}{l}\text { Porción del PIBE del sector industria manufac- } \\
\text { turera. INEGI (2009) }\end{array}$ \\
\hline & $\begin{array}{l}\text { Tamaño de las } \\
\text { empresas }\end{array}$ & $\begin{array}{l}\text { Razón de unidades económicas de tamaño } \\
\text { grande y micro de las industrias manufacture- } \\
\text { ras, comercio y servicios. Inegi (2009) }\end{array}$ \\
\hline \multirow{3}{*}{$\begin{array}{l}6 \text { Comunica- } \\
\text { ción externa e } \\
\text { internacionali- } \\
\text { zación de la } \\
\text { economía. }\end{array}$} & Comercio exterior & $\begin{array}{l}\text { Exportación más importación entre el PIB. } \\
\text { Secretaría de Economía (2008) }\end{array}$ \\
\hline & IED & $\begin{array}{l}\text { Inversión extranjera directa como porcentaje } \\
\text { del pIB. Secretaría de Economía (2008) }\end{array}$ \\
\hline & Acceso a internet & $\begin{array}{l}\text { Porcentaje de hogares con acceso a internet. } \\
\text { Inegi (2008) }\end{array}$ \\
\hline \multirow[t]{3}{*}{$\begin{array}{l}7 \text { Capacidad } \\
\text { de difusión }\end{array}$} & $\begin{array}{l}\text { Uso de compu- } \\
\text { tadoras }\end{array}$ & $\begin{array}{l}\text { Computadoras por cada } 100 \text { habitantes. INEGI } \\
\text { (2008) }\end{array}$ \\
\hline & $\begin{array}{l}\text { Cobertura de la } \\
\text { telefonía }\end{array}$ & $\begin{array}{l}\text { Líneas telefónicas fijas y móviles por cada } 100 \\
\text { habitantes. Cofetel (2008) }\end{array}$ \\
\hline & $\begin{array}{l}\text { Empresas con ISO } \\
9000\end{array}$ & $\begin{array}{l}\text { Empresas con ISO } 9000 \text { por cada millón de la } \\
\text { PEA. Conacyt (2011) }\end{array}$ \\
\hline 8 Innovación & Propiedad intelectual & $\begin{array}{l}\text { Propiedad intelectual (patentes, modelos de } \\
\text { utilidad y diseños) solicitadas por cada } 10,000 \\
\text { de la PEA. IMPI (2010) }\end{array}$ \\
\hline
\end{tabular}

Fuente: elaboración propia.

Dimensión 5: Estructura productiva. El trabajo seminal de Pavitt (1984) mostró que las características sectoriales de una economía afectan la dirección, naturaleza e intensidad de la innovación. Por ello resulta importante conocer la estructura de actividades de producción y exportación de los sectores con diferentes niveles de generación de conocimiento e $\mathrm{I}+\mathrm{D}$. Sin embargo, este tipo de información resulta difícil de obtener al nivel estatal en México, por lo que se consideraron los indicadores de valor agregado en actividades industriales y exportaciones, la porción del PIB estatal aportado por el sector manufacturero y la relación de unidades económicas grandes a las micro de las industrias de manufactura, comercio y servicios. El objetivo es describir las características del sector productivo de los estados mediante el tamaño e importancia económica de los sectores que más aportan a las actividades de cті.

Dimensión 6: Comunicación externa e internacionalización de la economía. Un factor clave para el funcionamiento y desarrollo de un sistema 
de innovación es su capacidad para establecer vínculos con el mercado externo y con otros sistemas. Las relaciones comerciales internacionales permiten una interacción que conlleva la adquisición de conocimiento nuevo o complementario, así como acceso a mercados externos. Para medir el nivel de esta interacción se utilizaron tres indicadores: la suma de exportaciones e importaciones como promedio del PIB, la inversión extranjera directa como porcentaje del PIB y el acceso a internet como porcentaje de los hogares en cada entidad. Los dos primeros miden el nivel de interacción con mercados externos, y el tercero refleja el potencial de la región para entablar comunicación fuera de sus límites geográficos.

Dimensión 7: Capacidad de difusión. Otro factor importante implica la circulación y distribución de conocimiento y nuevas tecnologías a diferentes partes del sistema. David y Foray (1995) plantearon el poder redistributivo del sistema de innovación como un factor importante para su funcionamiento. Este poder está enfocado en la colaboración y sus enlaces entre diversos actores del sistema. Si bien no existe un indicador o indicadores que midan esta actividad directamente, algunos proxy adecuados al respecto pueden ser los siguientes: las computadoras distribuidas entre la población, las líneas de teléfono fijas y móviles por habitantes y las empresas con ISO 9000 en proporción a la población económicamente activa. Los primeros dos indicadores reflejan la difusión de tecnologías de información y comunicación, mientras que el tercero revela la difusión de prácticas de innovación específicas dentro de la economía.

Dimensión 8: Innovación. Esta última dimensión tiene que ver con los resultados de las actividades de $\mathrm{I}+\mathrm{D}$. El número de solicitudes de instrumentos de propiedad intelectual (patentes, diseños industriales y modelos de utilidad) ha sido ampliamente utilizado en la literatura como proxy de la generación de innovaciones, ya que constituye un indicador de los desarrollos tecnológicos obtenidos en una economía. Aunque una patente no se puede considerar estrictamente una innovación, si es un buen indicador de desempeńo en actividades relacionadas directamente con la innovación.

Para todos los indicadores contemplados, los datos de PIB, densidad de población, gasto en educación per cápita y propiedad intelectual solicitada fueron transformados mediante $\log 10$. Por su parte, el índice de corrupción se transformó mediante el recíproco, para invertir el sentido de su interpretación, ya que a diferencia de los demás indicadores, éste se interpreta como "menor $=$ mejor". Todos los datos para los 24 indicadores se estandarizaron para eliminar efectos de magnitud y poder hacerlos comparables. Finalmente, los indicadores fueron agregados mediante suma y promedio en cada dimensión, dando como resultado ocho índices 
compuestos para cada SRI. A partir de estos indicadores compuestos se realizaron los análisis posteriores.

\subsection{Métodos}

Con el fin de identificar y desechar variables redundantes, primeramente se realiza un análisis de correlación de Pearson con los 24 indicadores originales, una vez seleccionados los definitivos, se realiza un análisis de conglomerados para las 32 entidades federativas del país a partir de los valores estandarizados de cada indicador que cubren las ocho dimensiones consideradas clave para los SRI. Posteriormente, se validan los resultados del análisis de conglomerados a través de una serie de análisis de varianza (ANOVA) inter-grupal de una vía. Finalmente, se representan gráficamente las dimensiones de los SRI para cada conglomerado, y se realiza una discusión de los resultados observados.

\subsubsection{Análisis de cluster}

El principio básico del análisis de cluster es identificar grupos homogéneos en una muestra, minimizando la variabilidad dentro del grupo y maximizándola entre grupos diferentes.

Específicamente, en este estudio se ha utilizado la técnica de clusters jerárquicos, que parte de conformar un grupo con los dos casos más similares y repitir este proceso hasta formar un grupo con todas las observaciones. Asimismo, se ha elegido el tipo de enlace Ward y se han probado dos medidas de distancia, la euclidiana y la Manhattan (también denominada de "block"), con la idea de hacer posible la comparación de resultados, toda vez que diferentes técnicas de aglomeración pueden generar diferentes clusters, incluso con los mismos elementos.

Esta comparación ha permitido valorar la robustez de la tipología obtenida para los SRI mexicanos. Los algoritmos de aglomeración se han corrido utilizando los indicadores que expresan el desarrollo de las ocho dimensiones consideradas clave para el nivel de desarrollo de los SRI. Los análisis realizados permitieron definir la "distancia euclidiana" como la que de manera más eficiente discriminó entre grupos y formó clusters con mayores diferencias. Por esta razón solamente se presentarán resultados para la estructura de grupos encontrada a partir de enlace Ward y distancia euclidiana. 


\subsubsection{Análisis de varianza (ANOVA)}

El ANOVA es un método estadístico para encontrar diferencias significativas entre las medias de una variable dependiente relacionada con una o más variables independientes. En el presente estudio se realizó un ANOVA intergrupal, donde cada dimensión de innovación fue una variable dependiente y los clusters constituyeron una variable independiente. La intención de estos análisis fue la de validar la estructura de clusters encontrada en el análisis previo.

\section{Resultados: Una propuesta de taxonomía de los SRI de México con base en sus condiciones, capacidades y resultados}

\subsection{La obtención de clusters de SRI}

Un análisis de correlación de Pearson, entre los indicadores al interior de cada dimensión, nos permitió identificar algunas variables que pudieran ser redundantes y provocar la presencia de colinealidad, lo cual podría afectar el subsecuente análisis de cluster. Dicho análisis nos llevó a prescindir de las variables PIB (Dimensión 1), índice de calidad de transparencia (Dimensión 2), formación bruta de capital fijo (Dimensión 3), publicaciones per cápita y población con estudios de posgrado (Dimensión 4) y computadoras por cada 100 habitantes (Dimensión 7). Finalmente, se realizó el análisis de cluster con las restantes 18 variables.

\section{Figura I \\ Dendrograma de aglomeración jerárquica con enlace Ward y distancia euclidiana}

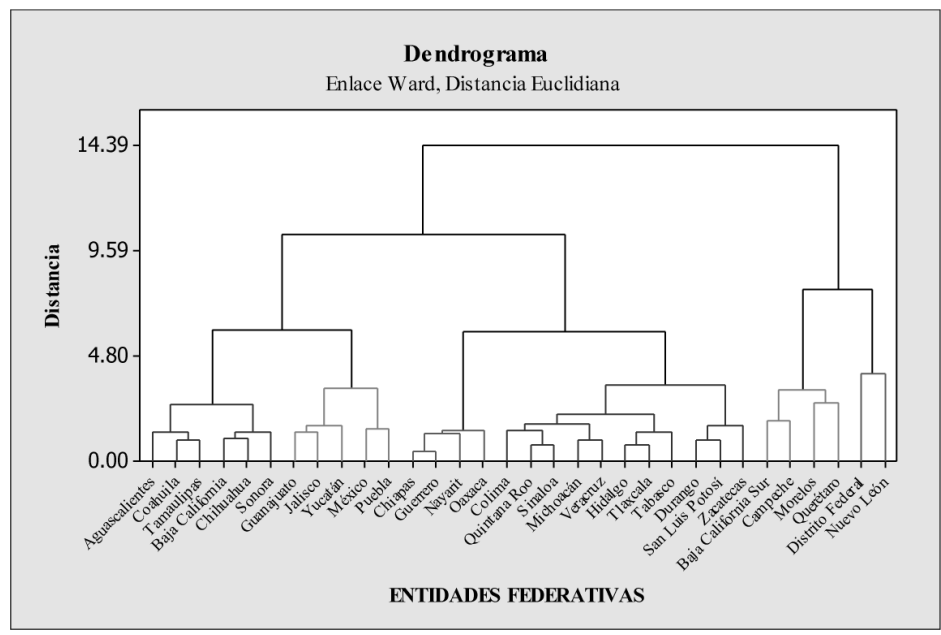

Fuente: elaboración propia mediante MINITAB16 ${ }^{\circ}$ Statistical Software. 
Partiendo del análisis de clusters jerárquico de enlace Ward y distancia euclidiana, la figura I muestra el dendrograma obtenido. Se observa claramente que cortando inmediatamente debajo del nivel de distancia de 4.80 se forman seis grupos distintivos. Los ANOVA realizados posteriormente dan sustento a la adecuada diferenciación de estos seis conglomerados, por lo cual sólo se presentarán los resultados obtenidos a partir de esta partición.

El cuadro 3 muestra los resultados del ANOVA realizado para comparar las medias entre los conglomerados por dimensión. Las diferencias son significativas, como se puede observar por los valores $p$ presentados en la tabla, lo cual sustenta la diferenciación entre conglomerados para la estructura encontrada.

\section{Cuadro 3 ANOVA intergrupal}

\begin{tabular}{|c|c|c|c|c|c|c|c|c|}
\hline \multicolumn{9}{|c|}{ Conglomerados } \\
\hline Dimensión & $\begin{array}{c}C 1 \\
(n=6)\end{array}$ & $\begin{array}{c}C 2 \\
(n=4)\end{array}$ & $\begin{array}{c}C 3 \\
(n=4)\end{array}$ & $\begin{array}{c}C 4 \\
(n=11)\end{array}$ & $\begin{array}{c}C 5 \\
(n=2)\end{array}$ & $\begin{array}{c}C 6 \\
(n=5)\end{array}$ & $P$ & $R 2 A j s$ \\
\hline $\begin{array}{l}\text { 1. Condiciones } \\
\text { de mercado }\end{array}$ & -0.0595 & -0.0905 & -0.6803 & -0.1706 & 2.2823 & 0.1505 & 0.000 & $58.22 \%$ \\
\hline $\begin{array}{l}\text { 2. Desarrollo } \\
\text { institucional }\end{array}$ & 0.0509 & -0.4900 & -0.8695 & 0.0085 & 0.2261 & 0.9173 & 0.003 & $38.58 \%$ \\
\hline $\begin{array}{l}\text { 3. Inversión en } \\
\text { intangibles y CF }\end{array}$ & 0.0059 & 0.3962 & -0.3930 & -0.1772 & 1.4159 & -0.1862 & 0.000 & $55.52 \%$ \\
\hline $\begin{array}{l}\text { 4. Conocimiento } \\
\text { científico }\end{array}$ & -0.2208 & 1.7732 & -0.6736 & -0.2094 & 0.7522 & -0.4549 & 0.000 & $67.58 \%$ \\
\hline $\begin{array}{l}\text { 5. Estructura } \\
\text { productiva }\end{array}$ & 0.8609 & -0.0989 & -1.0217 & -0.2482 & 0.6597 & 0.1455 & 0.000 & $56.09 \%$ \\
\hline $\begin{array}{l}\text { 6. Comunicación } \\
\text { externa }\end{array}$ & 0.9637 & -0.0615 & -0.8115 & -0.2561 & 0.8902 & -0.2506 & 0.000 & $61.19 \%$ \\
\hline $\begin{array}{l}\text { 7. Capacidad } \\
\text { de difusión }\end{array}$ & 0.2328 & 1.0862 & -1.0686 & -0.2766 & 1.9686 & -0.4725 & 0.000 & $80.83 \%$ \\
\hline 8. Innovación & -0.0390 & 0.1295 & -0.8388 & -0.5759 & 2.8757 & 0.7310 & 0.000 & $79.81 \%$ \\
\hline
\end{tabular}

\subsection{Las principales características de los distintos tipos de SRI}

Una vez que se han procesado los datos y conformado los clusters, se presentan los resultados para las ocho dimensiones de cada SRI (entidad) mediante gráficas de radar. Esta primera aproximación a la presentación 
de resultados es particularmente práctica, ya que permite visualizar de manera sencilla las fortalezas y debilidades de cada SRI en función de las dimensiones de innovación utilizadas.

Los gráficos de radar muestran la configuración de cada cluster de SRI, de acuerdo al desempeño en cada una de las ocho dimensiones (figuras II, III, IV, V, VI y VII). Cada eje de los gráficos varía alrededor del cero, siendo este valor equivalente a la media estandarizada para cada dimensión. Los gráficos muestran las fortalezas y debilidades en términos de las dimensiones de los SRI dentro del cluster al que pertenecen.

La figura II muestra que el grupo o cluster número 1 (C1) está integrado por seis SRI que destacan en tres dimensiones particulares: estructura productiva, comunicación externa, y capacidad de difusión. Por otra parte, se observa debilidad dentro del grupo para las dimensiones concernientes a la producción de conocimiento científico y condiciones de mercado. En términos de los niveles de las dimensiones, no se observa un líder evidente en el grupo; sin embargo, Aguascalientes, Baja California y Coahuila muestran los mayores promedios a través de las ocho dimensiones. En cambio, Sonora se encuentra rezagado del grupo, a pesar de ser una entidad con una de las fronteras norte más importantes del país, así como con una cantidad significativa de industria de media y alta tecnología.

Figura II

\section{Características de los SRI del cluster 1}

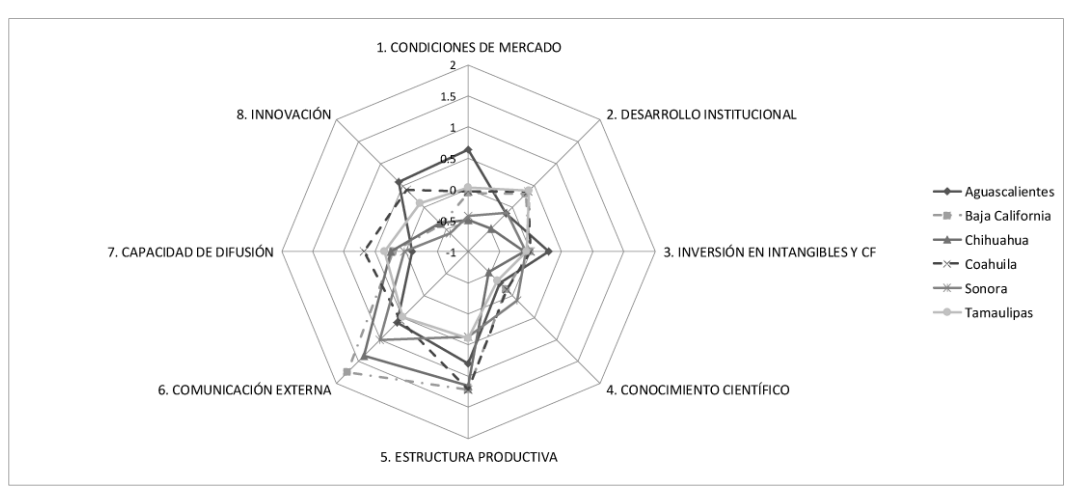

Fuente: elaboración propia mediante Microsoft Excel $2007^{\circ}$

Cabe destacar que este cluster está conformando principalmente por estados fronterizos, con excepción de Aguascalientes, donde se encuentra una importante industria maquiladora y otras industrias modernas de exportación, además de mantener una relación estrecha con EEUU debido 
a la frontera compartida. Es por eso que tiene sentido la predominancia de indicadores tales como exportaciones e importaciones, inversión extranjera directa, valor agregado en actividades industriales y exportaciones o incluso el porcentaje del PIB aportado por la industria manufacturera.

Entidades no fronterizas como Aguascalientes, también cuentan con una actividad industrial importante, y destacan en las dimensiones de estructura productiva y comunicación externa. En general, los resultados presentados por este cluster a través de las dimensiones de innovación lo posicionan en un nivel medio-alto, con especialización en estructura productiva y comunicación externa.

La figura III, corresponde al cluster número 2 (C2), mismo que muestra fortalezas en la capacidad para la generación efectiva y en la difusión de conocimiento científico, mientras que presenta debilidades en términos de su desarrollo institucional y su estructura productiva. Sin embargo, Querétaro contrasta con estos resultados, ya que presenta mejor desempeño en cuatro de las dimensiones (incluidas las dos anteriores), así como un mayor promedio general a través de las ocho dimensiones. Adicionalmente, se observa una irregularidad en las condiciones por dimensión de algunas entidades. Por ejemplo, Morelos presenta un valor alto en la generación de conocimiento científico en relación con las demás entidades. Campeche muestra un comportamiento similar, destacando en la dimensión de inversión en intangibles.

\section{Figura III \\ Características de los SRI del cluster 2}

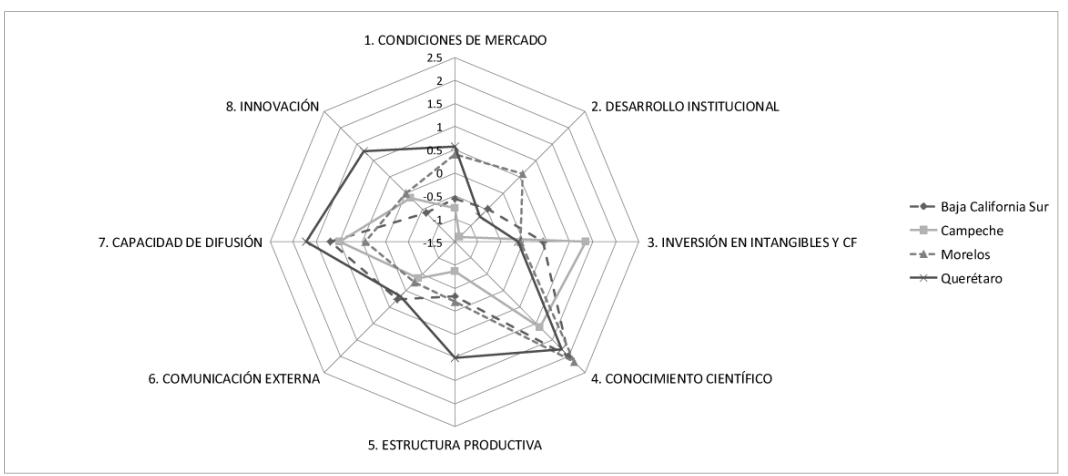

Fuente: elaboración propia mediante Microsoft Excel $2007^{\circ}$

Los integrantes de este grupo no se destacan por ser entidades con un gran desarrollo industrial o tecnológico, con la excepción de Querétaro. Las actividades económicas de estas entidades se enfocan más en actividades primarias, comercio y turismo. Se destaca el caso de Morelos para 
la dimensión de conocimiento científico compuesto por los indicadores de investigadores y centros de investigación en la entidad.

El valor relativamente alto de este indicador con respecto a los demás integrantes del grupo puede deberse a su cercanía con el centro del país (particularmente con el Distrito Federal), lo cual facilita la movilidad hacia instituciones de educación superior con un número importante de programas de posgrado. La entidad misma cuenta con 32 posgrados pertenecientes al Padrón Nacional de Posgrados de Calidad (PNPC), aunque la mayoría se concentran en áreas de humanidades y ciencias de la salud.

De manera general, el grupo tiende hacia el promedio del país en término de las dimensiones analizadas. $\mathrm{Al}$ igual que el cluster 1 , se observa un desempeño de nivel medio-alto, aunque con especialización en generación de conocimiento científico y difusión.

La característica más evidente del grupo de SRI que conforman el conglomerado 3 (ver figura Iv) es su rezago respecto a los sistemas del resto del país. Si bien todos los miembros de este grupo tienen condiciones económicas, tecnológicas y sociales, capacidades de innovación y resultados muy similares, destaca un desempeño relativo, en conjunto, muy pobre, ya que en casi todas las dimensiones de SRI, estas entidades se encuentran marcadamente debajo de la media.

\section{Figura IV \\ Características de los SRI del cluster 3}

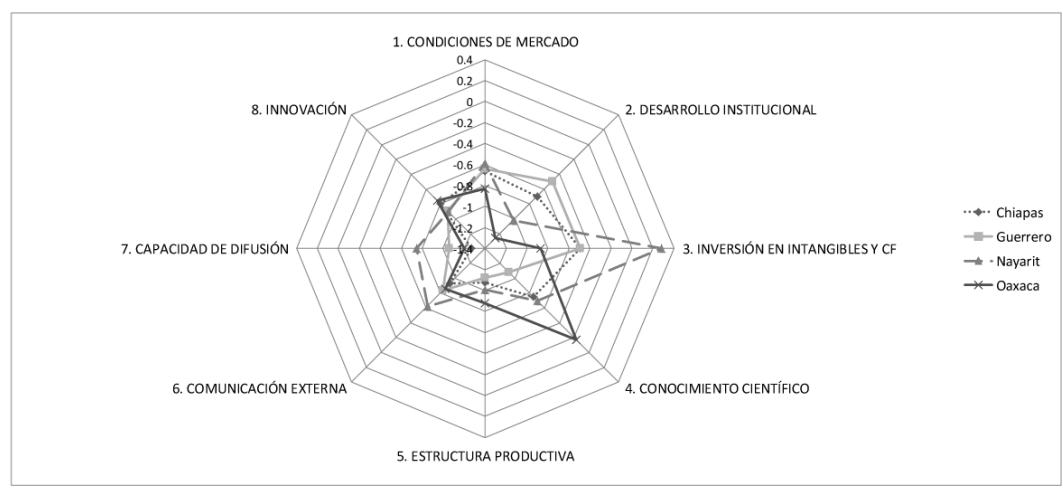

Fuente: elaboración propia mediante Microsoft Excel $2007^{\circ}$

Los bajos niveles en las diferentes dimensiones del grupo podría ser un indicativo de que no se cuentan con recursos adecuados para la conformación de sus sistemas. Históricamente, los estados que conforman este cluster han padecido un rezago importante en términos de desarrollo económico con respecto al resto del país. Finalmente, no destaca significativamente alguna entidad particular, aunque Nayarit exhibe un nivel 
sobre la media para la dimensión de inversión en intangibles. Sin embargo, el resto de sus dimensiones muestran los mismos niveles bajos que el resto de los integrantes del grupo. Debido a estas características, este cluster se caracteriza por un desempeño de nivel bajo.

El cluster 4 es el más numeroso, con 11 miembros en total. La gráfica de radar en la figura $\mathrm{V}$ muestra que, similar al grupo anterior, existe un rezago general a través de las dimensiones, aunque no al nivel del cluster 3. No se observa un líder entre las entidades que conforman el grupo, y en general la mayoría de las dimensiones presentan niveles por debajo de la media nacional para todos los miembros.

\section{Figura $v$}

\section{Características de los SRI del cluster 4}

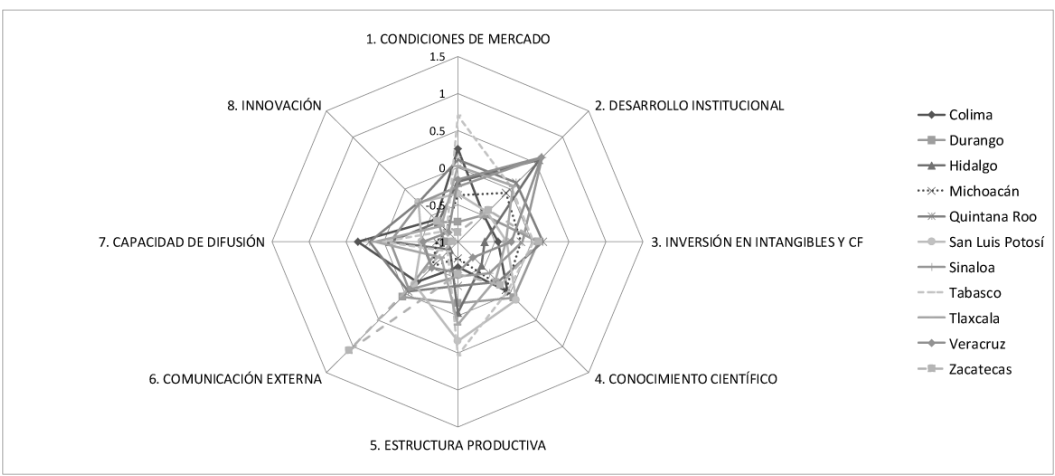

Fuente: elaboración propia mediante Microsoft Excel $2007^{\circ}$

El cluster 5 se encuentra conformado por dos entidades, Nuevo León y Distrito Federal. Ambos comparten la característica de tener los mejores niveles en las ocho dimensiones de análisis de los SRI de manera general. Es claro que el Distrito Federal es un sistema sui generis debido a la diferencia en su tamaño con respecto a cualquier otra entidad del país; no obstante, Nuevo León le aventaja notablemente en la dimensión de estructura productiva. En contraste, el Distrito Federal es significativamente superior bajo la dimensión de conocimiento científco. Es por eso que resulta interesante cuán nivelados se encuentran ambos sistemas en términos de la dimensión de innovación. Es posible que la producción de propiedad intelectual provenga de la investigación académica para el caso del Distrito Federal, mientras que para Nuevo León, la generación de pi se da mayormente a nivel empresarial.

En general, es evidente la superioridad de este grupo con respecto a los demás conglomerados y entidades individuales. Los niveles para la mayoría de sus dimensiones son significativamente superiores a los de 
cualquier otra entidad para las mismas dimensiones. Con la excepción de estructura productiva y tal vez, inversión en intangibles, el tamaño del Distrito Federal, en términos de su estructura organizacional e institucional, parece darle una ventaja importante sobre las demás entidades del país. Por mencionar algunos datos, esta entidad cuenta con 354 programas pertenecientes al PNPC, 6,606 investigadores en el Sistema Nacional de Investigadores, 376 instituciones de educación superior y 66 centros de investigación. Estos números muestran una gran diferencia, sólo en términos de su estructura de ciencia y tecnología, con respecto a las demás entidades. El Distrito Federal es, por sí sólo, el SRI más fuerte y consolidado del país. Sin embargo, el desempeño en términos del uso eficiente de sus recursos es una cuestión que amerita analizarse, pero que rebasa los objetivos de la presente investigación. Debido a las características anteriores, este cluster presenta un desempeño de nivel alto, con énfasis en sus condiciones de mercado, capacidad de difusión e innovación.

\section{Figura VI \\ Características de los SRI del cluster 5}

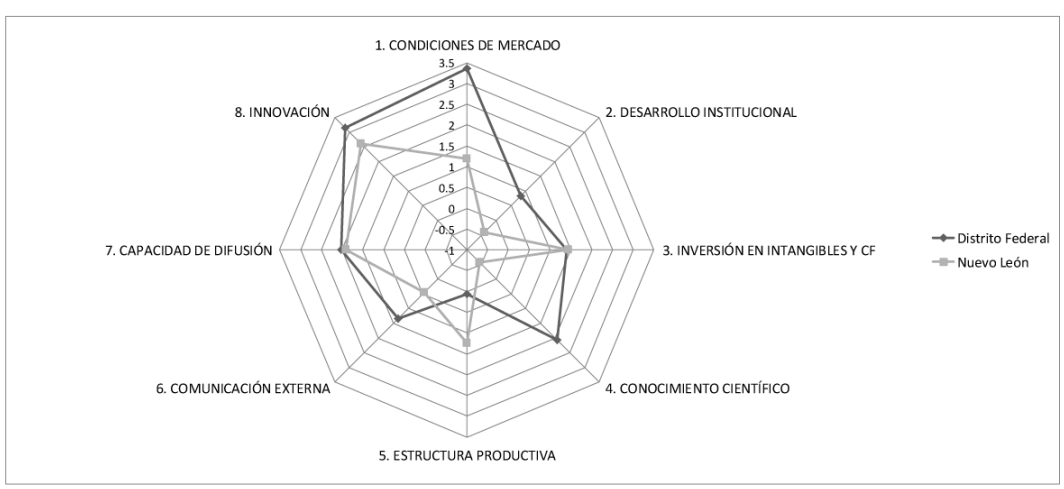

Fuente: elaboración propia mediante Microsoft Excel $2007^{\circ}$

Finalmente, el cluster 6 representado en la figura viI muestra sus ventajas en las dimensiones desarrollo institucional y resultados en innovación. Sin embargo, la inversión en intangibles, generación de conocimiento, comunicación externa y capacidad de difusión se encuentran por debajo de la media nacional. Esto representa 50\% de las dimensiones analizadas, lo cual es evidencia de SRI no consolidados. Por otra parte, el promedio de las dimensiones de cada integrante de este grupo se encuentra en niveles inferiores, por ejemplo, a los integrantes del conglomerado 1. Los integrantes más débiles del grupo son Puebla y Yucatán, quienes incluso muestran algunas características similares a los integrantes del cluster 4 . Todo lo anterior caracteriza al conglomerado 6 
por un desempeńo de nivel medio, destacando en lo particular en desarrollo institucional.

\section{Figura VII \\ Características de los SRI del cluster 6}

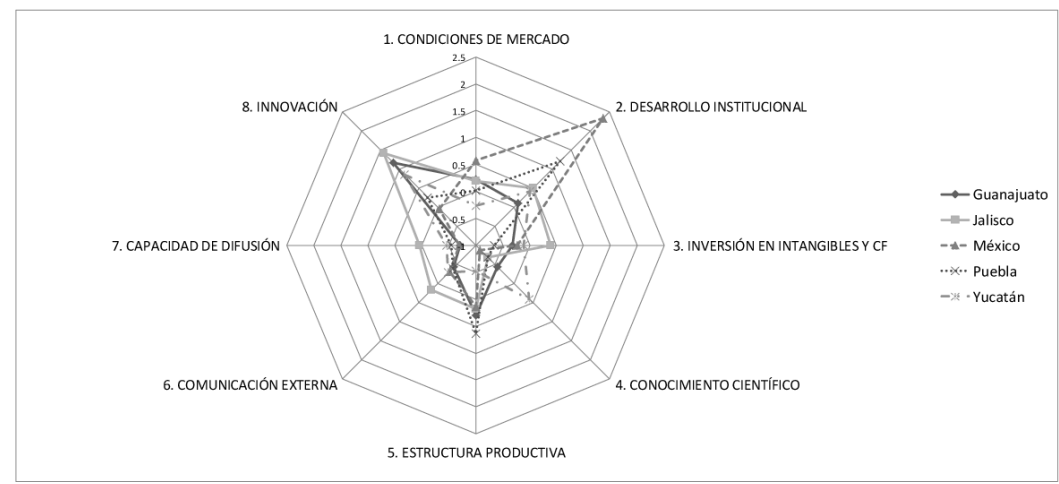

Fuente: elaboración propia mediante Microsoft Excel $2007^{\circledR}$

La información anterior permite discutir sobre el tamaño, homogeneidad y patrones en los SRI de México a partir de las dimensiones de los SRI propuestas. Subsecuentemente, nos es posible proponer una clasificación taxonómica a partir de estos resultados. El cuadro 4 describe las características particulares de cada tipo de SRI identificado en el análisis.

Estos resultados se asemejan a los obtenidos por los análisis de los SRI europeos, los cuales muestran una diversidad de configuraciones de sistemas, y proponen cinco o más tipologías (Clarysse y Muldur, 2001; Muller y Nauwelaers, 2005; Hollanders, 2007; Martínez-Pellitero et al., 2008). Asimismo, existen coincidencias con estudios como los realizados por Navarro y Gibaja (2009), también para el caso de regiones europeas, quienes encuentran que los clusters obtenidos se diferencian, en gran medida, con base en factores relacionados con las condiciones de desarrollo económico de las regiones y sus perfiles de especialización sectorial. Empero, en nuestro estudio, tales diferencias son explicadas, además, por otro tipo de precondiciones de la innovación, como el desarrollo institucional, la inversión en tangibles e intangibles y el grado de apertura externa de las regiones.

Otra semejanza consiste en que los SRI identificados por nuestro estudio presentan perfiles semejantes a los descritos en el estudio de Navarro et al. 2009, llevado a cabo en el contexto de la Unión Europea, quienes identifican SRI integrados por grupos con las siguientes características: a) regiones capital que cuentan en general con fuertes ventajas de aglome- 


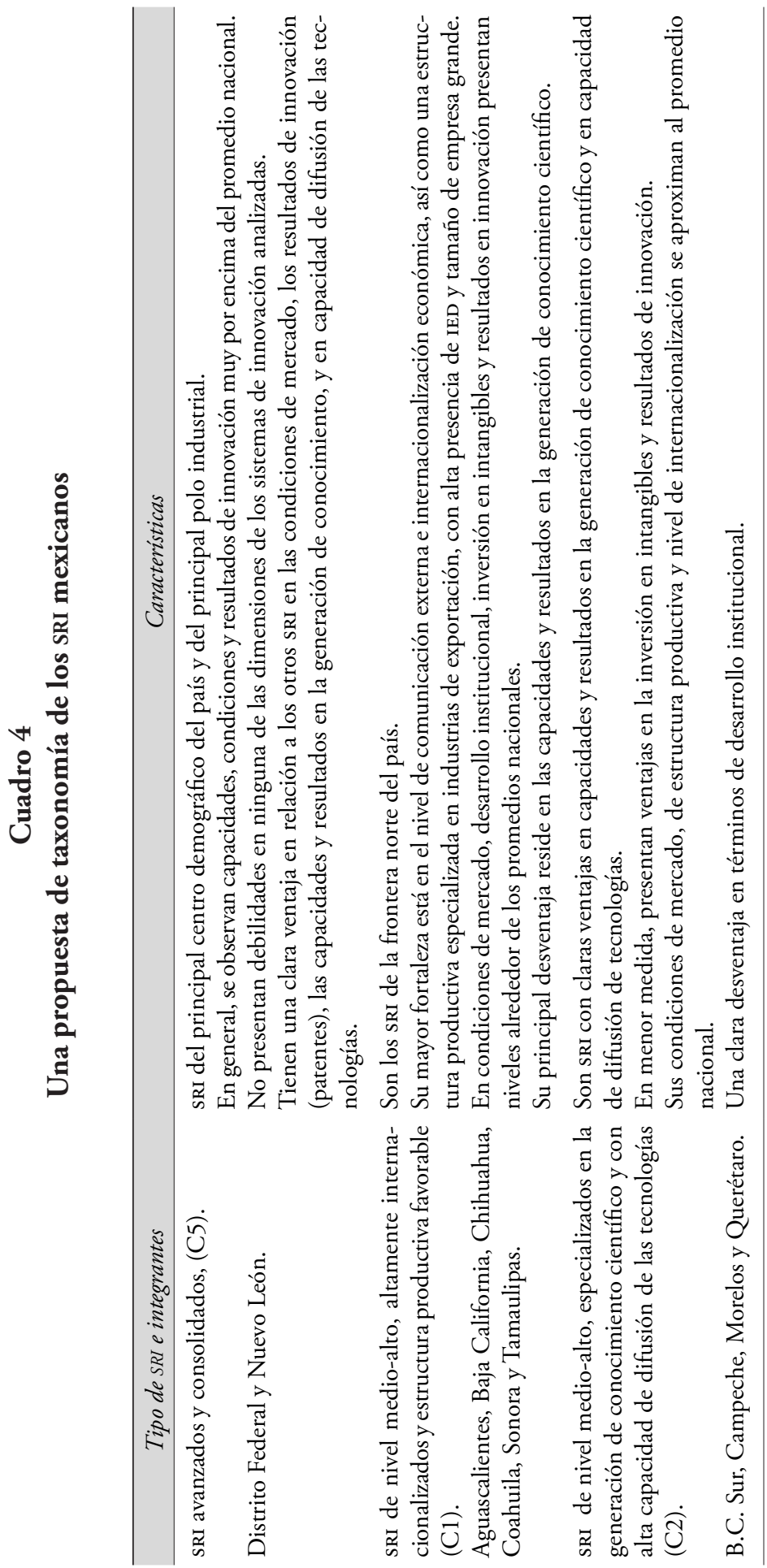




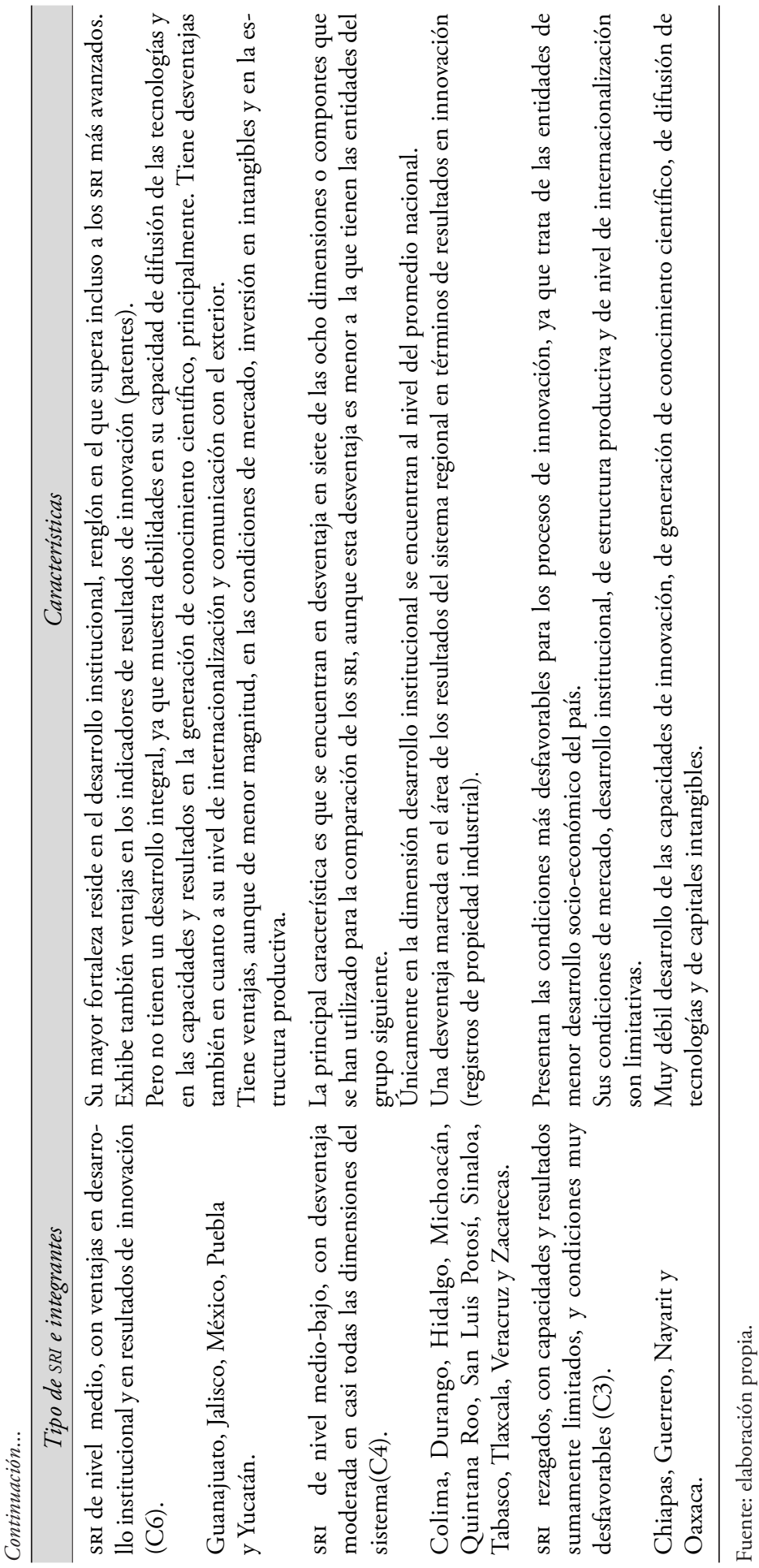


ración y servicios avanzados; b) regiones industriales especializadas en segmento de alta media tecnología y de exportación; c) regiones agropecuarias que cuentan con una débil infraestructura en CyT y capacidades muy limitadas para la difusión y generación del conocimiento.

La diferencia más importante, en este sentido, es que en los países más desarrollados un amplio grupo de los SRI identificados se concentra en el rango de las "regiones avanzadas tecnológicamente", mientras que en el caso de México, este grupo es muy reducido y predominan las "regiones rezagadas tecnológicamente" y los SRI categorizados de desarrollo intermedio-bajo.

Se ha identificado sólo un estudio que haya examinado las diferencias de los SRI mexicanos con una metodología similar a la empleada en este trabajo (Crespi y D’Este, 2011). Sin embargo, ambos difieren en la estructura dimensional y las variables empleadas para realizar el análisis de cluster, ya que el estudio mencionado se enfoca principalmente en los recursos públicos y privados para ciencia y tecnología, mientras que el nuestro hace uso, además, de variables que permitan describir condiciones y capacidades de los SRI. Por otra parte, si bien el estudio de Crespi y D'Este (2011) encuentra seis clusters al igual que nuestro estudio, no existe una intención evidente de proponer una clasificación taxonómica de los SRI, y la subsecuente caracterización de los grupos definidos es limitada con relación a lo realizado en nuestro estudio.

\section{Conclusiones}

El objetivo de este estudio fue construir una taxonomía de los sistemas regionales de innovación (SRI) que permita diferenciarlos con base en las características de capacidades, condiciones y desempeño, así como realzar una caracterización general de los tipos de SRI identificados. En particular, se utilizó como unidad de análisis cada una de las 32 entidades federativas de México y se recolectaron datos sobre los 24 indicadores agrupados en ocho grandes dimensiones, siguiendo el esquema de análisis propuesto por Godinho et al. (2006), que considera aspectos poco tratados en la literatura especializada sobre el tema, como las precondiciones para la innovación y los rasgos de las regiones, las entradas y salidas de los sistemas, entre otros

Los análisis de cluster y ANOVA arrojaron la conformación de seis grupos o tipos de SRI para el caso mexicano: i) SRI avanzados y consolidados; ii) SRI de nivel medio-alto, altamente internacionalizados y estructura productiva favorable; iii) SRI de nivel medio-alto, especializados en la generación de conocimiento científico y con alta capacidad de difusión de las tecnologías; iv) SRI de nivel medio, con ventajas en desarrollo ins- 
titucional y en resultados de innovación; v) SRI de nivel medio-bajo, con desventaja moderada en casi todas las dimensiones del sistema, y; vi) SRI rezagados, con capacidades y resultados sumamente limitados, y condiciones muy desfavorables.

Esta clasificación de los SRI mexicanos resulta útil para llevar a cabo una primera aproximación al análisis del nivel de diferenciación de las entidades federativas en términos de su potencial y desempeño para la innovación. Además, se realiza desde un nivel regional y evalúa la condición del país hacia el interior, sin necesidad de compararlo con otras naciones. Esto resulta relevante para países como México, que muestran niveles de desarrollo heterogéneos a través de sus diferentes regiones. En este sentido, aporta un nuevo enfoque para el estudio de los SRI para países en vías de desarrollo con características similares al caso mexicano.

Es importante mencionar que la propuesta se considera solamente un paso inicial para un análisis de las diferencias en la configuración de los SRI en México, el cual también debe incluir un análisis de eficiencia en el uso de recursos de ciencia, tecnología e innovación. La importancia de este tipo de estudios empíricos reside en la aportación que se pueda hacer a la consolidación del enfoque de sistemas de innovación como una herramienta práctica y legítima para explicar el desarrollo de regiones y naciones a partir de actividades de innovación. El fin último es poder generar una herramienta cuantitativa para la toma de decisiones en el área de política pública dedicada a los temas de ciencia, tecnología e innovación.

\section{Bibliografía}

Amable, Bruno (2003), The diversity of modern capitalism, Oxford University Press, Oxford.

Archibugi, Daniele (2001), "Pavitt's taxonomy sixteen years on: a review article", Economics of Innovation and New Technology, 10 (5), Taylor \& Francis, London, pp. 415-425.

Asheim, Björn y Philip Cooke (1999), "Local learning and interactive innovation networks in a global economy" en Edward Malecki y Paivi Oinas (eds.), Making connections: technological learning and regional economic change, Ashgate, Aldershot, pp. 145-178.

Asheim, Björn y Arne Isaksen (2002), "Regional innovation systems: the integration of local 'sticky' and global 'ubiquitous' knowledge", Journal of Technology Transfer, 1 (27), Springer, Austin, pp. 77-86. 
Asheim, Björn y Lars Coenen (2005), "Knowledge bases and regional innovation systems: Comparing Nordic clusters", Research Policy, 34 (8), Elsevier, Amsterdam, pp. 1173-1190.

Asheim, Björn y Meric Gertler (2006), “The geography of innovation. Regional innovation systems" en Jan Fagerberg, David Mowery y Richard Nelson (eds.), The Oxford Handbook of Innovation, Oxford University Press, Oxford, pp. 291-317.

Asheim, Björn (2007), "Sistemas regionales de innovación y bases de conocimiento diferenciadas: un marco teórico analítico", en Mikel Buesa y Joost Heijs (eds.), Sistemas regionales de innovación: nuevas formas de análisis y medición, Fundación de las Cajas de Ahorros, Madrid, pp. 65-89.

Balzat, Markus, y Andreas Pyka (2006), "Mapping national innovation systems in the OECD area", International Journal of Technology and Globalisation, 2 (1), Inder Science Publishers, Cambridge, pp. 158-176.

Buesa, Mikel, Joost Heijs, Mónica Martínez Pellitero y Thomas Baumert (2006), "Regional systems of innovation and the knowledge production function: the Spanish case", Technovation, 26 (4), Elsevier, London, pp. 463-472.

Campbelle, John y Ove Pedersen (2014), The national origins of policy ideas: knowledge regimes in the United States, France, Germany and Denmark, Princeton University Press, Princeton.

Capello, Roberta y Cailla Lenzi (2013), “Territorial patterns of innovation: a taxonomy of innovative regions in Europe", The Annals of Regional Science, 51 (1), Springer, Providence, pp. 119-154.

Capron, Henri y Michele Cincera (1998), The Flemish innovation system: an external viewpoint, IWT-Observation, Brussels.

CEesp (Centro de Estudios Económicos del Sector Privado, A. C.) (2008), "México: Calidad del Marco Regulatorio en las Entidades Federativas", <http://www.ceesp.org.mx/default.html>, 13 de agosto de 2012. 
CIEP (Centro de Investigación Económica y Presupuestaria) (2011), Gasto Público en Educación 2000-2010, Centro de Investigación Económica y Presupuestaria, México.

Clarysse, Bart y Ugur Muldur (2001), "Regional cohesion in Europe? An analysis of how EU public RTD support influences the technoeconomic regional landscape", Research Policy, 30 (2), Elsevier, Amsterdam, pp. 275-296.

Coccia, Mario (2006), "Classifications of innovations: Survey and future directions", CERIS working paper, Institute for Economic Research on Firms and Growth, Moncalieri.

Cofetel (Comisión Federal de Telecomunicaciones) (2008), Sistema de Información Estadística de Mercados de Telecomunicaciones (SIEMT), <http://siemt.cft.gob.mx/SIEM/>, 15 de agosto de 2012.

Comaip (Conferencia Mexicana para la Información Pública) (2008), "Métrica de la transparencia en México", Díaz Iturbe, Diego coord. (disponible en www.metricadetransparencia.cide.edu).

Conacyt (Consejo Nacional de Ciencia y Tecnología) (2011), Informe General del Estado de la Ciencia, la Tecnología y la Innovación, 2011, Consejo Nacional de Ciencia y Tecnología, México.

Cooke, Philip (1992), "Regional innovation systems: competitive regulations in the new Europe", Geoforum, 23 (3), Elsevier, London, pp. 365-382.

Cooke, Philip (1998), "Introduction. Origins of the concept", Regional Innovation Systems, UCL Press, London, pp. 2-25.

Cooke, Philip, Mikel Gomez-Uranga y Goio Etxebarria (1998), "Regional systems of innovation: an evolutionary perspective", Environment and Planning A, 30 (9), Pion Ltd, London, pp. 1563-1584.

Cooke, Philip, Patries Boekholt y Franz Tödtling (2000), The governance of innovation in Europe: Regional perspectives on global competitiveness, Pinter, London.

Crespi, Gustavo y Pablo D’Este (2011), “Análisis cuantitativo: la importancia del territorio en la conformación de los Sistemas Regio- 
nales de Innovación” en J.J. Llisterri y C. Pietrobelli (eds.), Los sistemas regionales de innovación en América Latina, Banco Interamericano de Desarrollo, Washington, pp. 28-57.

Crevoisier, Olivier, Roberto Camagni (2000), Les milieux urbains: innovation, systèmes de production et ancrage, EDES, Neuchâtel.

David, Paul y Dominique Foray (1995), "Accessing and expanding the science and technology knowledge base", STI Review, XVI, Organisation for Economic Co-operation and Development, Paris, pp. 13-68.

Diez-Revilla Javier (2002), "Metropolitan innovation systems: a comparison between Barcelona, Stockholm and Vienna", International Regional Science Review, 25 (1), Sage, Tempee, pp. 63-85.

Doloreux, David (2003), "Regional innovation systems in the periphery: The case of the Beauce in Québec (Canada)", International Journal of Innovation Management, 7 (1), World Scientific Publishing, Hackensack, pp. 67-94.

Doloreux, David y Saeed Parto (2004), Regional innovation systems: a critical synthesis, United Nations University-Institute for New Technologies, Maastricht.

Doty, Harold y William Glick (1994), “Typologies as a unique form of theory building: toward improved understanding and modeling", Academy of Management Review, 19 (2), Academy of Management, New York, pp. 230-251.

Ecotec (2005), The territorial impact of Eu research and development Policies, reporte para el proyecto ESPON 2.1.2, Birmingham.

Edquist, Charles, Marie-Louis Eriksson y Hans Sjögren (2000), “Collaboration in product innovation in the east Gothia regional system of innovation", Enterprise and Innovation Management Studies, 1 (1), Taylor \& Francis, Abingdon, pp. 37-56.

Enright, Michael (2003), "Regional clusters: what we know and what we should know" en Johannes Bröcker, Dirk Dohse y Rüdiger Soltwedel (eds.), Innovation clusters and interregional competition, Springer-Verlag, New York, pp. 99-129. 
Evangelista, Rinaldo, Tore Sandven, Giorgio Sirilli y Keith Smith (1998), "Measuring innovation in european industry", International Journal of the Economics of Business, 3 (5), Taylor and Francis, Abingdon, pp. 311-333.

Feldman, Maryann y David B. Audretsch (1999), "Innovation in cities: science-based diversity, specialization and localized competition", European Economic Review, 43 (2), Elsevier, Amsterdam, pp. 409-429.

Fссут (Foro Consultivo Científico y Tecnológico) (2010), Diagnósticos en Ciencia, Tecnología e Innovación, Foro Consultivo Científico y Tecnológico, México.

Fссут (Foro Consultivo Científico y Tecnológico) (2012), Diagnósticos en Ciencia, Tecnología e Innovación, Foro Consultivo Científico y Tecnológico, México.

ғссут (Foro Consultivo Científico y Tecnológico) (2014), Ranking Nacional de Ciencia, Tecnología e Innovación, capacidades y oportunidades de los sistemas estatales de ciencia, tecnología e innovación, Foro Consultivo Científico y Tecnológico, México.

Freeman, Christopher (1987), Technology policy and economic performance: lessons from Japan, Printer Publishers, London.

Gertler, Meric y David Wolfe (1998), "Dynamics of the regional innovation system in Ontario" en John de la Mothe y Gilles Paquet (eds.), Local and Regional Systems of Innovation, Kluwer Academic Publishers, Amsterdam, pp. 211-238.

Gertler, Meric, David Wolfe y David Garkut (2000), "No place like home? The embeddedness of innovation in a regional economy", Review of International Political Economy, 7 (4), Taylor \& Francis, Abingdon, pp. 688-718.

Godinho, Manuel Mira, Sandro Mendonça y Tiago Pereira (2006), “A taxonomy of national innovation systems: lessons from an exercise comprising a large sample of both developed, emerging and developing countries", ponencia presentada en la conferencia Globelics 2006, 4-7 de octubre, Kerala, India. 
Hall, Peter y David Soskice (2001), Varieties of capitalism: the institutional foundations of comparative advantage, Oxford University Press, Oxford.

Hollanders, Hugo (2007), European Regional Innovation Scoreboard (2006 RIS), MERIT, Maastricht.

Hudson, Ray (1999), "The learning economy, the learning firm and the learning region: a sympathetic critique of the limits to learning", European Urban and Regional Studies, 6 (1), Sage, London, pp. 59-72.

IMPI (Instituto Mexicano de la Propiedad Industrial) (2010), IMPI en cifras, <http:/www.impi.gob.mx/QuienesSomos/Paginas/IMPICifras.aspx>, 6 de marzo de 2012.

Inegi (Instituto Nacional de Estadística, Geografía e Informática) (2008), Banco de Información Económica <http://www.inegi.org.mx/ sistemas/bie/>, 8 de agosto de 2012.

Inegi (Instituto Nacional de Estadística, Geografía e Informática) (2009), Banco de Información Económica <http://www.inegi.org.mx/ sistemas/bie/>, 10 de agosto de 2012.

Inegi (Instituto Nacional de Estadística, Geografía e Informática) (2010), Banco de Información Económica <http://www.inegi.org.mx/ sistemas/bie/>, 8 de agosto de 2012.

Isaksen, Arne (2004), "Knowledge-intensive industries, clustering, and regional development. The software industry in Norway", Urban Studies, 41 (5-6), Sage, Glasgow, pp. 1157-1174.

Jacobides, Michael y Alina Kudina (2013), "How industry architectures shape firm success when expanding in emerging economies", Global Strategy Journal, 3 (2), Strategic Management Society, London, pp. 150-170.

Jang, Jae-Hong (2006), "Regional (sub-national) innovation system and the policy practice: the korean case", trabajo presentado en el National Workshop on Sub-national Innovation System and Technology Capacity Building Policies to Enhance Competitive- 
ness of Small and Medieum Enterpirses, 21-22 de diciembre, Katmandu, Nepal.

Latouche, Daniel (1998), "Do regions make a difference? The case of science and technology policies in Quebec" en Hans-Joachim , Philip Cooke y Martin Heindenreich (eds), Regional Innovation Systems: The Role of Governances in a Globalized World, UCL Press, London, pp. 319-344.

Lundvall, Bengt-Åke (1985), Product innovation and user-producer interaction, Aalborg University Press, Aalborg.

MacKinnon, Danny, Andrew Cumbers y Keith Chapman (2002), "Learning, innovation and regional development: a critical appraisal of recent debates", Progress in Human Geography, 26 (3), Sage, Glasgow, pp. 293-311.

Malmberg, Anders y Peter Maskell (1997), “Towards an explanation of regional specialization and industrial agglomeration”, European Planning Studies, 5 (1), Taylor \& Francis, Abingdon, pp. 25-41.

Martínez-Pellitero, Monica (2007), "Los sistemas regionales de innovación en Europa: tipología y eficiencia” (pp. 215-256), en Mikel Buesa y Joost Heijs (eds), Sistemas regionales de innovación: nuevas formas de análisis y medición, Madrid: Fundación de las Cajas de Ahorro.

Martínez-Pellitero, Mónica, Mikel Buesa, Joost Heijs y Tomas Baumert (2008), "A novel way of measuring regional systems of innovation: factor analysis as a methodological approach", documento de trabajo 60, Instituto de Análisis Industrial y Financiero, Madrid.

Muller, Emmanuel y Claire Nauwelaers (2005), "Enlarging the ERA: identifying priorities for regional policy focusing on research and technological development in the new members states and candidate countries", reporte final COP6-CT.2004.00001, Narcis, La Haya.

Navarro, Mikel y Juan José Gibaja (2009), “Typologies of innovation based on statistical analysis for european and spanish regions", documento de trabajo Innova, 2009-4, Madrid. 
Navarro, Mikel, Juan José Gibaja, Beñat Bilbao-Osorio y Ricardo Aguado (2009), "Patterns of innovation in EU-25 regions: a typology and policy recommendations", Environment and Planning C: Government and Policy, 27 (5), Pion Ltd, Londres, pp. 815-840.

Niosi, Jorge (2002), "National systems of innovations are 'x-efficient' (and x-effective): Why some are slow learners", Research Policy, 31 (2), Elsevier, Amsterdam, pp. 291-302.

OECD (Organización para la Cooperación y Desarrollo Económicos) (1992), Proposed guidelines for collecting and interpreting technological innovation data: Oslo Manual, Organización para la Cooperación y Desarrollo Económicos, Paris.

Pavitt, Keith (1984), "Sectoral patterns of technical change: towards a taxonomy and a theory", Research Policy, 13 (6), Elsevier, London, pp. 343-373.

Pinto, Hugo y Tiago Santos (2013), "Efficiency of innovation systems in Europe: an institutional approach to the diversity of national profiles", European Planning Studies, 21 (6), Taylor \& Francis, Abingdon, pp. 755-779.

Porter, Michael (1998), "Clusters and the new economics of competition”, Harvard Business Review, Harvard Business Publishing, Cambridge, pp. 77-90.

Reslinger, Coralie (2014), "Is there an Asian model of technological emergence?”, Socio-Economic Review, 11 (2), Oxford University Press, Oxford, pp. 371-408.

Santos, Domingos y Maria João Simōes (2014), "Regional innovation systems in Portugal: a critical evaluation", Investigaciones Regionales, 28, Marcial Pons, Madrid, pp. 37-56.

Saxenian, AnnaLee (1994), Regional advantage. Culture and competition in silicon valley and route 128, Harvard University Press, Cambridge.

Secretaría de Economía (2008), Dirección General de Inversión Extranjera, <http://www.economia.gob.mx/comunidad-negocios/ 
competitividad-normatividad/inversion-extranjera-directa>, 10 de septiembre de 2012.

Simmie, James (2001), Innovative cities, Spon Press, London.

Smith, Keith (2005), "Measuring Innovation" en Jan Fagerberg, David Mowery y Richard Nelson (eds.), The Oxford handbook of innovation, Oxford University Press, Oxford, pp. 148-177.

Transparencia Mexicana (2007), "Índice Nacional de Corrupción y Buen Gobierno", <http://www.tm.org.mx/wp-content/uploads/2013/ 05/01-INCBG-2010-Informe-Ejecutivo1.pdf> , 2 de septiembre de 2012.

Warriner, Charles (1984), Organizations and their environments. Essays in the sociology of organizations, Jai Press, Greenwich.

Recibido: 20 de diciembre de 2012.

Reenviado: 22 de octubre de 2013.

Aceptado: 11 de diciembre de 2013.

Cuitláhuac Valdez-Lafarga. Mexicano. Es estudiante de doctorado en ciencias, especialidad en desarrollo regional, en el Centro de Investigación en Alimentación y Desarrollo, A. C. (CIAD) en Hermosillo, Sonora. Su línea de investigación es desarrollo regional e innovación tecnológica.

Jorge Inés León-Balderrama. Mexicano. Es doctor en ciencias sociales por la Universidad Autónoma de Sinaloa. Actualmente es investigador titular en la Coordinación de Desarrollo Regional del Centro de Investigación en Alimentación y Desarrollo, A. C. (CIAD) en Hermosillo, Sonora, y profesor de los programas de maestría en desarrollo regional y doctorado en ciencias del CIAD. Es miembro del Sistema Nacional de Investigadores, nivel I. Sus líneas de investigación actuales son: desarrollo regional e innovación tecnológica, transferencia de tecnología y relaciones academia-empresa. Entre sus últimas publicaciones destacan, en coautoría: "Transferencia de conocimiento y cambio organizacional en las instituciones de I+D de Sonora, México", Innovation/Innovación/InovaçãoRICEC, 3 (2), RICEC, Montreal, pp.1-22 (2012);; "El CIAD y el mejoramiento de la competitividad de las Mipyme locales de la industria de alimentos: evaluación de la efectividad del Programa de Servicios Estratégicos", Estudios Sociales, número especial 2; vol. XXX, CIAD A. C. 
Hermosillo, pp. 97-123 (2012); "La formación de cuerpos académicos en México. Desarrollo e influencia en la producción de conocimiento (2002-2009)" en Luis Huesca-Reynoso, Mario-Camberos Castro y Cuauhtémoc Calderón: Bienestar y desarrollo en el siglo XXI, Plaza y Valdez-CIAD, México, pp. 207- 220 (2011). 